ka auszuschleichen, sei dagegen nicht evidenzbasiert und eher ökonomischen Überlegungen zuzuschreiben, so Krüger. Dieses Vorgehen entspreche nicht dem Wunsch der Patienten, die Methotrexat (MTX) als erstes absetzen möchten. Hierfür sprechen auch mehrere Studien und Daten des niederländischen DREAM-Registers, die zeigen konnten, dass MTX ohne Wirkverlust vor Biologika abgesetzt werden kann. Krüger riet, die Entscheidung individuell gemeinsam mit dem Patienten zu treffen.

Beim Ausschleichen der Biologika sei zu beachten, dass es sich bei einer Dosisreduktion oder Intervallverlängerung zumindest bei den subkutanen Anwen- dungen um eine Off-label-Therapie handele. Ein Regress sei aber nicht zu befürchten.

rheuma plus $2017 \cdot 16: 124-126$ https://doi.org/10.1007/s12688017-0145-z

๑) Springer-Verlag GmbH Austria 2017

\title{
JIA-Therapieoptionen oft zu spät genutzt
}

Auch für die juvenile idiopathische Arthritis gilt, dass dem Kinderrheumatologen inzwischen eine breite Palette, selbst an Biologika, zur Verfügung steht. Es sollte unbedingt versucht werden, die Krankheitsaktivität innerhalb des ersten Jahres unter Kontrolle zu bringen.

Seit dem Jahr 2000, als Etanercept als erstes Biologikum für die polyartikuläre juvenile idiopathische Arthritis (JIA) für Kinder ab zwei Jahren zugelassen wurde, sind Adalimumab, Tocilizumab und Golimumab hinzugekommen, letzteres mit Einschränkung für ein Gewicht von $>40 \mathrm{~kg}$. Werden die Therapieziele mit diesen Tumornekrosefaktor Alpha-Inhibitoren (TNFI) nicht erreicht, kann Abatacept eingesetzt werden, allerdings erst bei Kindern ab sechs Jahren. Dabei müssen Abatacept und Golimumab laut Zulassung mit Methotrexat (MTX) kombiniert werden, wie Prof. Dr. Gerd Horneff, Zentrum für Allgemeine Pädiatrie und Neonatologie, Kinderrheumazentrum Sankt Augustin, Asklepios Klinik Sankt Augustin, auf dem Kongress der DGRh in der Sitzung JJuvenile idiopathische Arthritis -Was gibt es Neues?" in Erinnerung rief.

\section{Früher Biologika-Einsatz gerade bei der SJIA}

Auch für andere JIA-Kategorien wurden inzwischen eine Reihe von Biologika geprüft und zugelassen. Für die Enthesitis-assoziierte JIA (ERA) kann Adalimumab bei Kindern ab sechs Jahren und Etanercept neben der ERA auch bei der Psoriasis-Arthritis von Jugendlichen ab zwölf Jahren eingesetzt werden. Bei der systemischen JIA (sJIA), dem Morbus Still, stehen mit Canakinumab und Tocilizumab ab einem Alter von zwei Jahren zwei Biologika zur Auswahl.

Trotz dieser Therapieoptionen vergeht im klinischen Alltag laut Horneff immer noch zu viel Zeit bis effektive Medikamente eingesetzt werden. Die Krankheitsaktivität bestehe im Median mehr als zwei Jahre. Die rasche Kontrolle innerhalb des ersten Erkrankungsjahres sei aber für das LangzeitOutcome von großer Bedeutung, wie in einer deutschen Kohortenstudie gezeigt werden konnte. Horneff rief daher dazu auf, sich enger an die Therapieleitlinien zu halten und einen Treat-to-Target-Ansatz mit regelmäßiger Kontrolle der Krankheitsaktivität und entsprechender Eskalation der Therapie zu verfolgen.

\section{\) Trotz der Therapieoptionen vergeht im klinischen Alltag immer noch zu viel Zeit bis zum Einsatz effektiver Medikamente}

Gerade beim Morbus Still, bei dem häufig weder MTX noch TNFI zum gewünschten Erfolg führen, sollte die Therapie früh eskaliert werden. Denn
Kortikosteroide können aufgrund der induzierten Wachstumsstörungen und Osteoporose bzw. Osteopenie nur begrenzt eingesetzt werden. Insofern werde heute eine den Empfehlungen des American College of Rheumatology (ACR) entsprechende frühe, an der Pathogenese orientierte, steroidfreie Therapie mit Interleukin 1 (IL-1)- oder IL-6-Hemmern favorisiert.

D) Trotz dieser Therapieoptionen vergeht im klinischen Alltag immer noch zu viel Zeit bis effektive Medikamente eingesetzt werden

Als Innovationen für die Therapie der JIA sind laut Horneff zunächst weitere Biologika in Form von anderen IL-6-Inhibitoren wie Sarilumab und IL-17AInhibitoren wie Secukinumab zu erwarten. Zudem befinden sich als orale Therapieoptionen die beiden Janus-Kinase-Inhibitoren Baricitinib und Tofacitinib in der klinischen Prüfung.

rheuma plus $2017 \cdot 16: 126$ https://doi.org/10.1007/s12688017-0142-2

(c) Springer-Verlag GmbH Austria 2017 